\title{
POSTER
}

\section{Les blocs osseux allogéniques en chirurgie pré- implantaire : une revue de la littérature}

\author{
Borie $\mathbf{G}^{1,2}$, Lamure $\mathbf{J}^{1,2}$, Le Roy $\mathbf{R}^{1,2}$, Paul $\mathbf{A}^{1,2}$
}

1 - CHU de Nice, Hôpital Saint-Roch, Pôle Odontologie, 5 rue Pierre Dévoluy, 06000 Nice, France

2 - UFR d'Odontologie de Nice, UNS, 24 avenue des Diables Bleus, 06000 Nice, France

\section{Introduction}

La réhabilitation prothétique supra-implantaire nécessite une qualité et un volume osseux suffisant pour la mise en place des implants dans des conditions biologiques et mécaniques optimales afin de garantir une stabilité du résultat fonctionnel et esthétique. Lorsque ce volume n'est pas suffisant, une chirurgie osseuse pré-implantaire est nécessaire. L'autogreffe est reconnue comme étant le " gold standard " dans les techniques de greffes osseuses pré-implantaires. Cependant, la faible quantité d'os en site donneur chez certains patient, la nécessité d'un second site opératoire, l'augmentation du temps d'intervention et la morbidité associée au prélèvement nous amènent à trouver des solutions alternatives.

Les substituts osseux sont une alternative à l'os autogène. L'allogreffe est la principale alternative à ce jour : c'est la greffe de tissu d'un individu à un autre d'une même espèce. Elle présente des propriétés évidentes d'ostéoconduction, cependant, ses propriétés ostéoinductrices sont encore sujet à controverse. Le but de cette étude est de faire le point sur les possibilités d'augmentations de volume ainsi que sur le taux de survie implantaire des implants posés dans ces augmentations osseuses.

\section{Matériels et méthode}

Une recherche sur Pubmed d'articles concernant l'utilisation des blocs allogéniques en chirurgie pré-implantaire a été réalisée. Les mots clés utilisés étaient : « block allograft dental », " corticocancellous allogeneneic dental », « fresh-frozen allogeneic ». Sur 77 articles répertoriés, la lecture du résumé a permis une sélection de 41 articles. Enfin, une lecture intégrale a permis d'inclure au final 23 articles pertinents dans cette étude.

L'analyse de la littérature concernant l'utilisation des allogreffes en bloc indique une ré-intervention pour la phase implantaire à 4-6 mois. L'ensemble des articles étudiés nous montre un taux de survie implantaire à plus de $95 \%$, ce qui est similaire à l'os non greffé.

La moyenne des augmentations osseuses est de $2-3 \mathrm{~mm}$ en vertical et de $5-7 \mathrm{~mm}$ en horizontal, ce qui, avec les diamètres et les surfaces implantaires actuelles, permet de répondre à la majorité des situations cliniques. La littérature conclut à une alternative fiable aux greffes autogènes.

Le taux de survie des blocs allogéniques est de $93 \%$ à 12 mois. Dans $69 \%$ des cas, aucune résorption n'est observée. Une faible résorption, inférieure à 1-2 $\mathrm{mm}$ et limitée à une faible surface, est observée dans les autres cas. Aucune résorption majeure n'est rapportée dans la littérature odontologique. Quelques cas de résorption ont néanmoins été rapportés dans la 
littérature de chirurgie orthopédique. Concernant la chirurgie pré-implantaire, le suivi est faible et, contrairement à l'autogreffe et à la xénogreffe, nous ne disposons pas, à l'heure actuelle, d'un recul suffisant.

La littérature nous rapporte des pourcentages d'os vital dans les greffes osseuses allogéniques en bloc variant de $62 \%$ à $33 \%$.

L'étude de Schmitt en 2012, compare le pourcentage d'os vital des carottes osseuses de greffes sinusiennes autogènes, allogènes, xénogènes et alloplastiques. Les greffes autologues ont un pourcentage d'os vital de $42 \%$, les allogreffes de $35 \%$, les allogreffes de $30 \%$ et les xénogreffes $25 \%$.

\section{Conclusion}

Les greffes osseuses par blocs allogéniques sont une réelle alternative aux greffes autogènes pour des greffes horizontales. Elles seront particulièrement indiquées chez des patients ne souhaitant ou ne pouvant bénéficier de greffes autogènes.

L'utilisation de procédés CAD/CAM se développe, permettant de réduire le temps opératoire et les risques de fracture lors de la fixation du bloc. 